\title{
Encuesta sobre el control de la anemia en pacientes con enfermedad renal crónica en diálisis en Latinoamérica
}

\author{
María N. Campistrús ${ }^{1 *}$, Raúl G. Carlini², Liliana Andrade ${ }^{3}$, Liliana Chifflet ${ }^{4}$, María E. F. Canziani ${ }^{5}$, \\ Gregorio T. Obrador ${ }^{6}$, Hugo Poblete ${ }^{7}$ y Alberto Locatelli ${ }^{8}$
}

${ }^{1}$ Comité de Educación Médica Continua, Sociedad Uruguaya de Nefrología, Montevideo, Uruguay; ${ }^{2}$ Servicio de Nefrología y Trasplante Renal, Hospital Universitario de Caracas, Caracas, Venezuela; ${ }^{3}$ Hospital Churruca Visca (PFA), Buenos Aires, Argentina; ${ }^{4}$ Fondo Nacional de Recursos, Montevideo, Uruguay; ${ }^{5}$ Disciplina de Nefrología, Universidad Federal de São Paulo, São Paulo, Brasil; ${ }^{6}$ Facultad de Ciencias de la Salud y Escuela de Medicina, Universidad Panamericana, Ciudad de México, México; ${ }^{7}$ Centro de Diálisis Sermedial, Valparaíso, Chile; ${ }^{8}$ Nefrología Argentina, Buenos Aires, Argentina. En representación del Comité de Anemia de la Sociedad Latinoamericana de Nefrología e Hipertensión (SLANH)

\begin{abstract}
Resumen
El tratamiento de la anemia de los pacientes en diálisis en Latinoamérica se conoce apenas parcialmente. Esta encuesta explora los criterios diagnósticos y terapéuticos para controlar la anemia de dicha población, así como los resultados alcanzados y las dificultades enfrentadas. Métodos: Encuesta por internet, voluntaria, anónima, dirigida a médicos que asisten a pacientes en hemodiálisis crónica (HDC) o diálisis peritoneal (DP) en Latinoamérica. Exploramos los criterios para usar agentes estimulantes de eritropoyesis (AEE) y hierro intravenoso (Fe/V), los exámenes disponibles, la hemoglobina ( $\mathrm{Hb}$ ) deseada, los resultados terapéuticos y las dificultades percibidas. Resultados: Respondieron 513 médicos de 20 países: el $75.2 \%$ eran nefrólogos; el 77.5\% habían trabajado $\geq 5$ años en diálisis; el $71.3 \%$, en HDC, y el $31.6 \%$, en DP. El $84.4 \%$ determinaban la $\mathrm{Hb}$ mensualmente. Los parámetros ferrocinéticos más usados fueron la ferritina $(79.1 \%)$ y la saturación de transferrina (73.1\%), aunque con una disponibilidad limitada. Más del 75\% de los pacientes usaban AEE, según el 79.8\% de los encuestados, mientras que ese porcentaje recibía FelV según el $46.5 \%$. Los criterios para indicar AEE fueron variados, así como el nivel deseado de $\mathrm{Hb}$. Sólo el $54 \%$ de los encuestados refirieron $\geq 75 \%$ de pacientes tratados con AEE en un rango de $\mathrm{Hb}$ deseada, mientras que el $14.7 \%$ refirieron $>25 \%$ de pacientes con $\mathrm{Hb}$ baja $\sin \mathrm{AEE}$. El $74 \%$ reconocieron las limitaciones para el tratamiento asociadas con un inadecuado control de la anemia. Conclusiones: Se evidenció un uso más frecuente de AEE que de FelV. Hubo criterios disímiles para prescribir AEE, definir la $\mathrm{Hb}$ deseada y monitorear el tratamiento. Apenas la mitad de los encuestados logró un control satisfactorio de la Hb con AEE. Las limitaciones percibidas se asociaron con anemia mal controlada.
\end{abstract}

Palabras clave: Anemia. Hemodiálisis. Diálisis peritoneal. Encuesta. Latinoamérica.

\section{Anemia control survey in Latin American end stage renal disease patients}

\section{Abstract}

The treatment of anemia in Latin American dialysis patients is partially known. We performed a survey to evaluate the criteria used for the diagnosis and treatment of anemia in this population, to examine the achievement of therapeutic targets and

\section{Correspondencia:}

*María Nieves Campistrús

E-mail: mncampist@gmail.com

DOI: 10.24875/NEFRO.18000015
Disponible en internet: 28-05-2018

Nefro Lat. 2018;15:16-28

www.nefrologialatinoamericana.com 2444-9032/@ 2018 Sociedad Latinoamericana de Nefrología e Hipertensión. Publicado por Permanyer México SA de CV. Este es un artículo Open Access bajo la licencia CC BY-NC-ND (http://creativecommons.org/licenses/by-nc-nd/4.0/). 
difficulties faced by clinicians. Methods. A voluntary and anonymous on-line survey was sent to physicians who take care of patients receiving chronic hemodialysis (HDC) or peritoneal dialysis (PD) in Latin America. We investigated the criteria to prescribe erythropoiesis stimulating agents (ESAs) and intravenous iron (FelV), available laboratory tests, target hemoglobin $(\mathrm{Hb})$, therapeutic achievements and perceived difficulties for anemia control. Results. 513 physicians from 20 countries replied to the survey: $75.2 \%$ were nephrologists, $77.5 \%$ have worked $\geq 5$ years on dialysis, $71.3 \%$ in HDC and $31.6 \%$ in PD. Hb was measured monthly by $84.4 \%$ of responders. The more common ferrocinetic parameters were ferritin $(79.1 \%)$ and transferrin saturation (73.1\%), but their availability was limited. More than $75 \%$ of patients received ESA and FeIV according to $79.8 \%$ and $46.5 \%$ of responders respectively. The indications for prescription of ESA were diverse, as it was the target Hb level. Only $54 \%$ of the responders stated that $\geq 75 \%$ of patients treated with ESA were in the Hb target. Likewise, $14.7 \%$ reported $>25 \%$ of patients with low $\mathrm{Hb}$ was not receiving treatment. Lastly, $74 \%$ of responders acknowledged several limitations for the treatment of anemia. Conclusions. ESA was more often used than FelV. There were dissimilar criteria regarding ESA prescription, $\mathrm{Hb}$ target, and treatment monitoring. Several limitations affected anemia control.

Key words: Anemia. Hemodialysis. Peritoneal dialysis. Survey. Latin America.

\section{Introducción}

El manejo de la anemia en la enfermedad renal crónica (ERC) constituye un pilar fundamental del tratamiento, dado que su diagnóstico oportuno y control adecuado se asocian a mejor calidad de vida y probablemente a mayor sobrevida de esos pacientes ${ }^{1,2}$. La prevalencia y severidad de la anemia son mayores a medida que el filtrado glomerular decrece, y son máximas al llegar el paciente a la etapa de ERC avanzada, cuando requiere un tratamiento sustitutivo ${ }^{3,4}$.

En Latinoamérica, la epidemiología, los métodos diagnósticos y las posibilidades de tratamiento de la anemia de pacientes en diálisis apenas se conocen en base a datos procedentes de estudios parciales. Éstos muestran una gran heterogeneidad entre los diferentes países y poblaciones consideradas. Registros previos, tanto nacionales como multinacionales, muestran que, pese al extenso uso de AEE, los resultados terapéuticos suelen ser pobres, y que existe una gran variabilidad entre países ${ }^{5,6}$.

El Comité de Anemia de la Sociedad Latinoamericana de Nefrología e Hipertensión (SLANH) trabaja desde 2007 con el objetivo de promover el adecuado tratamiento de la anemia en pacientes con ERC de nuestro continente a fin de reducir la morbimortalidad y mejorar su calidad de vida con una buena relación costo/efectividad. El comité elaboró las Recomendaciones SLANH para el tratamiento de la anemia en el paciente con enfermedad renal crónica y publicó su primer informe sobre prevalencia, estudio y tratamiento de la anemia de pacientes en HDC de Latinoamérica ${ }^{7}$. En ese estudio participaron 154 nefrólogos de 16 países que aportaron datos válidos de 9,025 pacientes, correspondientes al $4 \%$ de la población total en diálisis, según el Registro Latinoamericano de Diálisis y Trasplante (RLADT) ${ }^{8}$. Se halló que sólo un tercio de esos pacientes presentaban $\mathrm{Hb}$ en el rango recomendado por este comité (10.5$12.0 \mathrm{~g} / \mathrm{dl})$, y que, pese al extenso uso de AEE (84.3\%), cerca de la mitad de los pacientes tenían $\mathrm{Hb}$ inferior a dicho rango.

Con el fin de complementar dicho estudio, se decidió realizar una encuesta entre colegas latinoamericanos que tratan a pacientes en diálisis crónica, procurando aumentar el número de participantes utilizando un cuestionario sencillo y rápido de responder. El objetivo de la encuesta fue explorar los criterios diagnósticos y terapéuticos para controlar la anemia de los pacientes en diálisis en Latinoamérica, así como los resultados alcanzados y las dificultades percibidas.

\section{Métodos}

Se trata de un estudio transversal basado en una encuesta estandarizada, con un mínimo de respuestas abiertas. Se preguntó sobre la percepción de aspectos vinculados al tratamiento de la anemia en pacientes en diálisis. La encuesta estaba dirigida a nefrólogos y médicos de América Latina que asistían a pacientes en HDC o DP, y se administró por internet, en español y portugués. La convocatoria se realizó por correo electrónico. La directiva de la SLANH la envió a todos sus miembros, así como a las autoridades de las sociedades locales que la integran, las cuales difundieron la encuesta entre sus respectivos socios. Estuvo activa desde el 1 de diciembre de 2013 hasta el 31 de marzo de 2014. Las respuestas fueron voluntarias y anónimas, sin recompensa para los participantes. El cuestionario primario fue elaborado en español por uno de los autores y enviado a los demás miembros del Comité de Anemia de la SLANH para su revisión. Con base en las opiniones recibidas, se 
adecuaron algunas de las preguntas, y el cuestionario se reenvió a los mismos colegas. Una vez alcanzado el consenso, se tradujo al portugués. Con 21 preguntas de diversos formatos se exploró lo siguiente: edad, sexo, país, ámbito laboral, nivel académico y experiencia, criterios para usar FelV y $\mathrm{AEE}$, valor de $\mathrm{Hb}$ deseado y alcanzado, estudios de laboratorio habituales $(\mathrm{Hb}, \mathrm{Hb}$ corpuscular media, volumen corpuscular medio, sideremia, índice de saturación de transferrina [IST], ferritina sérica, contenido de $\mathrm{Hb}$ reticulocitaria, porcentaje de glóbulos rojos hipocrómicos y «otros»), así como las dificultades que enfrentaban los encuestados para controlar la anemia de sus pacientes en diálisis (Anexo 1).

Otros aspectos explorados fueron: tipo de institución donde se trabaja, número de pacientes asistidos y tipo de tratamiento del agua para hemodiálisis.

Las preguntas referentes al porcentaje de pacientes tratados con AEE o FelV proponían cuatro opciones de respuesta: más del $90 \%$, entre el 75 y el $90 \%$, entre el 50 y el $75 \%$, y menos del $50 \%$. También se categorizaron las respuestas referentes a valores de $\mathrm{Hb}$, ya fuera el usado para indicar $\mathrm{AEE}$, ya fuera el máximo deseado.

Se definió «buen control» si $\geq 75 \%$ de pacientes tratados con AEE estaban en el rango de $\mathrm{Hb}$ deseada $y$ «mal control» cuando ese resultado se alcanzaba en menos del $75 \%$ de pacientes.

Las preguntas sobre el porcentaje de pacientes con estudios de laboratorio ofrecían cuatro categorías de respuesta: más del $80 \%$, entre el 40 y el $80 \%$, menos del $40 \%$ y ninguno.

Algunas preguntas daban opción para responder "Otros", así como para escribir comentarios.

Las dificultades para estudiar y/o tratar la anemia se clasificaron según tres áreas diferentes: limitaciones para evaluar el perfil férrico, para administrar AEE y para administrar FelV. Cada área presentaba opciones para identificar el tipo de dificultad: alto costo para el paciente, alto costo para el centro de diálisis, dificultades burocráticas para obtener recursos de las instituciones pública o privada que financian la asistencia, no adherencia del paciente a las indicaciones y ninguna limitación.

Respecto al análisis estadístico, se utilizó un paquete estadístico estándar (SPSS para Windows, versión 17.0; SPSS, Chicago, IL). Los datos se analizaron como variables cualitativas caracterizadas por frecuencia y proporción. Para evaluar las asociaciones entre las categorías de respuesta y el grado de control de la anemia, se realizó un análisis bivariado con la prueba de chi cuadrada de Pearson. La asociación era significativa si $p<0.05$.

Esta encuesta se realizó manteniendo el anonimato y protección de datos personales de los participantes.

\section{Resultados}

\section{Población encuestada}

La encuesta fue respondida en forma válida, aunque no siempre completa, por 513 médicos procedentes de 20 países de Latinoamérica. Sus características demográficas y distribución por país se muestran en la tabla 1. El $87.1 \%$ de los encuestados eran nefrólogos o residentes en Nefrología y el $77.5 \%$ de ellos tenían más de cinco años de experiencia asistiendo a pacientes en diálisis. La mayoría asistía fundamentalmente a pacientes en HDC, mientras que el $31.6 \%$ trataban a pacientes en DP.

\section{Tratamiento de la anemia de los pacientes en diálisis crónica}

La tabla 2 muestra la frecuencia de uso de AEE y FelV. Se observa que 373 encuestados $(200+173$; $79.8 \%$ ) respondieron que el $75 \%$ o más de sus pacientes recibían AEE. En relación con el FelV, se recibieron 462 respuestas, el $46.5 \%$ de las cuales refirieron su uso en $\geq 75 \%$ de los pacientes asistidos; 102 encuestados $(22.1 \%)$ lo daban a menos del $50 \%$ de su población.

Los criterios para indicar AEE y el valor máximo de $\mathrm{Hb}$ deseado con ese tratamiento se presentan en la tabla 3.

La tabla 4 muestra la distribución de las respuestas según el grado de compensación de la anemia con AEE. Se preguntó qué porcentaje de pacientes tratados con AEE se hallaban en el rango de $\mathrm{Hb}$ esperado: más del $75 \%$ de pacientes tratados con $A E E$ estaban en el rango de $\mathrm{Hb}$ deseada según el $54 \%$ de los encuestados, constituyendo el grupo de «buen control». Con igual criterio, el grupo con menos del $75 \%$ de pacientes tratados con AEE en valores de $\mathrm{Hb}$ deseada («mal control») estuvo constituido por el $46 \%$ de los encuestados $(n=215)$. No existe diferencia según se atienda predominantemente HDC o DP. Asimismo, la frecuencia de reportes de «buen control» 0 «mal control» no fue diferente según el objetivo de $\mathrm{Hb}$ planteado, ni según la cualidad de los encuestados de ser o no nefrólogos. Los médicos que utilizaban AEE o FeIV en un porcentaje de 
Tabla 1. Características de la población encuestada y distribución según los países $(n=513)$

\begin{tabular}{|c|c|c|}
\hline Participantes & $\mathbf{n}$ & $\%$ \\
\hline $\begin{array}{l}\text { Género } \\
\text { Masculino }\end{array}$ & 289 & 56.3 \\
\hline $\begin{array}{l}\text { Edad } \\
\text { Hasta } 30 \text { años } \\
\text { De } 31 \text { a } 40 \text { años } \\
\text { De } 41 \text { a } 50 \text { años } \\
\text { Más de } 50 \text { años }\end{array}$ & $\begin{array}{c}23 \\
152 \\
140 \\
198\end{array}$ & $\begin{array}{r}4.5 \\
29.6 \\
27.3 \\
38.6\end{array}$ \\
\hline $\begin{array}{l}\text { Nivel académico* } \\
\text { Doctor en Medicina } \\
\text { Residente de Medicina } \\
\text { Residente de Nefrología } \\
\text { Nefrólogo }\end{array}$ & $\begin{array}{c}71 \\
18 \\
61 \\
386\end{array}$ & $\begin{array}{c}13.8 \\
3.5 \\
11.9 \\
75.2\end{array}$ \\
\hline $\begin{array}{l}\text { Experiencia en diálisis crónica }(n=487) \\
\text { Menos de } 1 \text { año } \\
\text { De } 1 \text { a } 5 \text { años } \\
\text { De } 5 \text { a } 10 \text { años } \\
\text { Más de } 10 \text { años }\end{array}$ & $\begin{array}{c}7 \\
103 \\
105 \\
272\end{array}$ & $\begin{array}{c}1.4 \\
21.1 \\
21.6 \\
55.9\end{array}$ \\
\hline $\begin{array}{l}\text { Área principal de trabajo* } \\
\text { Nefrología clínica } \\
\text { HDC } \\
\text { DP } \\
\text { Trasplante } \\
\text { Investigación } \\
\text { Administración }\end{array}$ & $\begin{array}{l}327 \\
366 \\
162 \\
90 \\
37 \\
32\end{array}$ & $\begin{array}{l}63.7 \\
71.3 \\
31.6 \\
17.5 \\
7.2 \\
6.2\end{array}$ \\
\hline $\begin{array}{l}\text { Tipo de institución donde trabaja* } \\
\text { Hospital universitario } \\
\text { Hospital público (no universitario) } \\
\text { Hospital privado } \\
\text { Centro de diálisis independiente } \\
\text { Centro de empresa trasnacional }\end{array}$ & $\begin{array}{c}112 \\
166 \\
95 \\
191 \\
73\end{array}$ & $\begin{array}{l}21.8 \\
32.4 \\
18.5 \\
37.2 \\
14.2\end{array}$ \\
\hline $\begin{array}{l}\text { Procedencia (país) } \\
\text { Argentina } \\
\text { México } \\
\text { Chile } \\
\text { Perú } \\
\text { Venezuela } \\
\text { Brasil } \\
\text { Uruguay } \\
\text { Colombia } \\
\text { Ecuador } \\
\text { Bolivia } \\
\text { Guatemala } \\
\text { El Salvador } \\
\text { Paraguay } \\
\text { República Dominicana } \\
\text { Costa Rica } \\
\text { Cuba } \\
\text { Honduras } \\
\text { Nicaragua } \\
\text { Panamá } \\
\text { Puerto Rico }\end{array}$ & $\begin{array}{l}77 \\
73 \\
64 \\
48 \\
48 \\
46 \\
40 \\
28 \\
27 \\
11 \\
11 \\
10 \\
10 \\
7 \\
4 \\
3 \\
2 \\
2 \\
1 \\
1\end{array}$ & $\begin{array}{c}15.0 \\
14.2 \\
12.5 \\
9.4 \\
9.4 \\
9.0 \\
7.8 \\
5.5 \\
5.3 \\
2.1 \\
2.1 \\
1.9 \\
1.9 \\
1.4 \\
0.8 \\
0.6 \\
0.4 \\
0.4 \\
0.2 \\
0.2\end{array}$ \\
\hline
\end{tabular}

*Algunos participantes marcaron más de una opción.

pacientes menor no reportaron una frecuencia mayor de «mal control» que aquéllos que los usaban en $\geq$ $75 \%$ de su población.
Tabla 2. Respuestas a la pregunta sobre el porcentaje de pacientes que reciben AEE y FeIV

\begin{tabular}{|l|c|c|}
\hline $\begin{array}{l}\text { Tipo de respuesta } \\
\text { Porcentaje de pacientes que } \\
\text { usan }\end{array}$ & \multicolumn{2}{|c|}{ N. ${ }^{\circ}$ de respuestas (\%) } \\
\hline Más del $90 \%$ & $85(18.4)$ & $200(42.8)$ \\
\hline Entre el 75 y el $90 \%$ & $130(28.1)$ & $173(37)$ \\
\hline Entre el 50 y el $75 \%$ & $145(31.4)$ & $71(15.2)$ \\
\hline Menos del $50 \%$ & $102(22.1)$ & $23(4.9)$ \\
\hline N. ${ }^{\circ}$ total de respuestas & 462 & 467 \\
\hline No responden & $51(9.9)$ & $46(8.9)$ \\
\hline
\end{tabular}

Tabla 3. Criterios para indicar AEE y valor máximo de $\mathrm{Hb}$ deseada

\begin{tabular}{|l|c|c|}
\hline \multirow{2}{*}{$\begin{array}{l}\text { Usted indica AEE a sus pacientes } \\
\text { en diálisis cuando la Hb }\end{array}$} & \multicolumn{2}{|c|}{ Respuestas } \\
\cline { 2 - 3 } & $n$ & $\%$ \\
\hline $11 \mathrm{~g} / \mathrm{dl}$ & 118 & 25.11 \\
\hline $10.5 \mathrm{~g} / \mathrm{dl}$ & 89 & 18.94 \\
\hline $10 \mathrm{~g} / \mathrm{dl}$ & 173 & 36.8 \\
\hline $9.5 \mathrm{~g} / \mathrm{dl}$ & 74 & 15.74 \\
\hline Ninguno de los anteriores & 16 & 3.4 \\
\hline Total de respuestas & 470 & 100 \\
\hline No responden & 43 & 8.3 \\
\hline ¿Cuál es el máximo valor de Hb & Respuestas \\
\hline que usted pretende al tratar a sus & $n$ & $\%$ \\
\hline $11 \mathrm{~g} / \mathrm{dl}$ & 114 & 23.5 \\
\hline $12 \mathrm{~g} / \mathrm{dl}$ & 259 & 53.4 \\
\hline $13 \mathrm{~g} / \mathrm{dl}$ & 75 & 15.5 \\
\hline Ninguno de los anteriores & 7 & 1.4 \\
\hline Variable para cada paciente & 30 & 6.2 \\
\hline Total de respuestas & 485 & 100 \\
\hline No responden & 28 & 5.4 \\
\hline
\end{tabular}

Al preguntar sobre qué porcentaje de pacientes portadores de $\mathrm{Hb}$ inferior al rango deseado no reciben eritropoyetina (u otro AEE), se recibieron 467 respuestas, que se detallan en la tabla 5. Destaca que, para el $14.7 \%$ de los encuestados, más del $25 \%$ de los pacientes con $\mathrm{Hb}$ inferior a la deseada no recibían AEE. 
La determinación de $\mathrm{Hb}$ se realizaba con una frecuencia variable: mensualmente según el $84.4 \%$ de los encuestados; el $7.9 \%$ la medían cada dos meses; el $5.3 \%$, cada tres meses, y el $2.4 \%$ respondieron que se determinaba sin periodicidad. La ferritina sérica $(79.1 \%)$ y el IST (73.1\%) fueron los estudios ferrocinéticos más frecuentemente solicitados, pero durante el último año sólo el $68.7 \%$ de los encuestados disponían de IST y el $75.7 \%$ refirieron contar con ferritina en más del $80 \%$ de sus pacientes. El contenido de $\mathrm{Hb}$ reticulocitaria y el porcentaje de glóbulos rojos hipocrómicos estaban disponibles para el 3.9 y el $4.9 \%$ de los encuestados, respectivamente.

\section{Dificultades para cumplir con el tratamiento}

Ante la pregunta «¿Qué tipo de limitaciones enfrenta usted para disponer de los recursos básicos para tratar la anemia de sus pacientes en diálisis?», 133 encuestados $(28 \%)$ respondieron «Ninguna limitación». Los demás refirieron uno o más tipos de dificultades. La tabla 6 resume los diferentes tipos de limitaciones reconocidas.

Entre los encuestados que no refirieron limitaciones de ningún tipo, 87 (65.4\%) expresaron que más del $75 \%$ de los pacientes tratados con AEE estaban en el rango de $\mathrm{Hb}$ deseado, mientras que 165 de los que refirieron algún tipo de limitación (49.4\%) consiguieron ese «buen control» (chi cuadrada: 9.817; $p=$ 0.002). Asimismo, entre los 149 encuestados que expresaron que el estudio y/o tratamiento de la anemia significaba un «alto costo para el paciente», sólo $64(43 \%)$ refirieron que más del $75 \%$ de los pacientes con AEE lograban la $\mathrm{Hb}$ deseada, mientras que el $59 \%$ de los colegas que no citaban esa dificultad lograban ese «buen control» con AEE (chi cuadrada: 10.49; $p=0.001$ ).

El reconocimiento de las limitaciones del estudio y tratamiento de la anemia también se asocia con las respuestas referentes al porcentaje de pacientes con $\mathrm{Hb}$ baja que no recibían AEE. Entre los 133 encuestados que refirieron no tener ninguna limitación para estudiar y tratar la anemia, 94 (71\%) expresaron que todos sus pacientes con $\mathrm{Hb}$ bajo el rango deseado recibían AEE y sólo $39(29 \%)$ reconocieron que había pacientes con $\mathrm{Hb}$ baja que no los recibían. La mayoría $(64.7 \%)$ de los 334 encuestados que refirieron algún tipo de limitación reconoció tener pacientes con $\mathrm{Hb}$ baja que no recibían AEE (chi cuadrada: 46.864; $p=0.000$ ).
Tabla 4. Frecuencia con que se alcanza el objetivo de tratamiento con AEE

\begin{tabular}{|l|c|c|}
\hline $\begin{array}{l}\text { ¿Qué porcentaje de sus pacientes } \\
\text { tratados con AEE se hallan en el } \\
\text { rango de Hb esperado? }\end{array}$ & \multicolumn{2}{|c|}{ Respuestas } \\
\cline { 2 - 3 } & n & $\%$ \\
\hline Más del $90 \%$ & 39 & 8.4 \\
\hline Entre el 75 y el $90 \%$ & 213 & 45.6 \\
\hline Entre el 50 y el 75\% & 171 & 36.6 \\
\hline Menos del $50 \%$ & 44 & 9.4 \\
\hline Total de respuestas & $\mathbf{4 6 7}$ & $\mathbf{1 0 0}$ \\
\hline No responden & 46 & 8.9 \\
\hline
\end{tabular}

Tabla 5. Frecuencia de respuestas que refieren pacientes portadores de $\mathrm{Hb}$ inferior al rango deseado que no reciben $\mathrm{AEE}$

\begin{tabular}{|l|c|c|}
\hline \multirow{2}{*}{$\begin{array}{l}\text { Tipo de respuesta (porcentaje de } \\
\text { pacientes) }\end{array}$} & \multicolumn{2}{|c|}{ Respuestas } \\
\cline { 2 - 3 } & n & $\%$ \\
\hline Más del $50 \%$ & 24 & 5.1 \\
\hline Entre el 25 y el $50 \%$ & 45 & 9.6 \\
\hline Menos del 25\% & 186 & 39.8 \\
\hline Ninguno & 212 & 45.4 \\
\hline Total de respuestas & $\mathbf{4 6 7}$ & $\mathbf{1 0 0}$ \\
\hline No responden & 46 & 8.9 \\
\hline
\end{tabular}

Tabla 6. Dificultades referidas para el estudio y/o tratamiento de la anemia de los pacientes en diálisis crónica en Latinoamérica

\begin{tabular}{|l|c|c|}
\hline Tipo de dificultades & No de respuestas & $\%$ \\
\hline Alto costo para el paciente & 149 & 31.4 \\
\hline $\begin{array}{l}\text { Alto costo para el centro de } \\
\text { diálisis }\end{array}$ & 117 & 24.7 \\
\hline $\begin{array}{l}\text { Burocráticas con institución } \\
\text { pública }\end{array}$ & 201 & 42.4 \\
\hline $\begin{array}{l}\text { Burocráticas con institución } \\
\text { privada }\end{array}$ & 86 & 18.1 \\
\hline No adherencia del paciente & 120 & 25.3 \\
\hline Sin limitaciones para & 176 & 37.1 \\
\hline Evaluar el capital férrico & 167 & 35.2 \\
\hline Administrar AEE & 173 & 36.5 \\
\hline Administrar FelV & $\mathbf{1 3 3}$ & $\mathbf{2 8}$ \\
\hline Ninguna limitación & $\mathbf{4 7 4}$ & 7.6 \\
\hline Total de respuestas & 39 & \\
\hline Sin respuesta & & \\
\hline
\end{tabular}




\section{Análisis cualitativo de los comentarios opcionales recibidos}

Entre los colegas encuestados, 157 emitieron comentarios al final de la encuesta. Todos fueron pertinentes con el tema de la encuesta y coherentes con las respuestas brindadas. Se abordaron múltiples aspectos, que se resumen en la tabla 7 .

Según 24 comentarios, la ausencia de limitaciones se vinculaba a que la medicación era suministrada por el sistema sanitario o estaba incluida en el precio de la diálisis; aun así, varios referían dificultades para realizar estudios de laboratorio.

También se expresaron críticas a la encuesta: no se consultaba sobre las dosis de AEE o FelV $(n=3)$, ni sobre la frecuencia de transfusiones de sangre $(n=2)$, y cinco colegas destacaron la importancia de considerar el estado nutricional del paciente en relación con el control de la anemia. Algunos colegas plantearon sugerencias: analizar la anemia de la etapa prediálisis $(n=3)$, definir el nivel adecuado de $\mathrm{Hb}$ en pacientes que viven a más de $2,500 \mathrm{~m}$ de altitud $(\mathrm{n}=2)$ y realizar cursos en línea sobre DP, HDC y anemia de la ERC $(n=3)$.

\section{Discusión}

Los principales hallazgos de esta encuesta expresan los variados criterios y logros terapéuticos, así como las frecuentes limitaciones para controlar la anemia de los pacientes en diálisis crónica en Latinoamérica. Tres cuartas partes de los médicos encuestados refirieron que el $75 \%$ o más de sus pacientes en diálisis recibían AEE; el uso de FelV resultó mucho menos frecuente. Los criterios para indicar AEE fueron disímiles. El objetivo de $\mathrm{Hb}$ a alcanzar con ese tratamiento resultó variable. También el logro del objetivo terapéutico con AEE fue heterogéneo, y cabe destacar que apenas el $54 \%$ de los encuestados refirieron que más del $75 \%$ de sus pacientes tratados habían alcanzado la $\mathrm{Hb}$ deseada, situación definida como «buen control» a los efectos de este estudio. Observados los índices de calidad y metas a alcanzar de distintas sociedades científicas, consideramos adecuado plantear un estándar de calidad intermedio para controlar la anemia en relación con otros de la región, y no demasiado exigente, por lo cual definimos «buen control» si $\geq 75 \%$ de la población tratada con AEE se hallaba con $\mathrm{Hb}$ deseada ${ }^{9,10}$.

La encuesta también evidencia criterios diversos para controlar la anemia. La $\mathrm{Hb}$ se determinaba mensualmente según el $84.4 \%$ de los encuestados; los demás la medían con menor frecuencia. Los parámetros ferrocinéticos más usados fueron la ferritina sérica (79.1\%) y/o el IST (73.1\%), pero su disponibilidad real resultó limitada.

Los criterios para indicar el tratamiento con AEE, la definición de $\mathrm{Hb}$ objetivo y la periodicidad para medir la $\mathrm{Hb}$ y los parámetros férricos estaban claramente establecidos en las guías de práctica clínica (GPC) vigentes en el momento de la encuesta y eran accesibles a todo médico, por su idioma y/o por hallarse disponibles en internet. Todas las guías recomendaban medir mensualmente la $\mathrm{Hb}$, lo cual no cumplía el $15 \%$

Tabla 7. Resumen de los comentarios opcionales recibidos ( $\mathrm{n}$ total $=157$ )

Dificultades burocráticas y/o altos costos para obtener medicación o realizar exámenes de laboratorio

Se brindan servicios heterogéneos entre la población (inequidad)

Escasa adhesión al tratamiento por parte del paciente/familia

Dudas sobre la eficacia de genéricos de eritropoyetina

Agradecen o felicitan por la realización de la encuesta

Se destacan otros problemas vinculados al control de la anemia en diálisis

Malnutrición y pobreza

Infecciones y procesos inflamatorios crónicos

Hiperparatiroidismo secundario mal controlado

Incumplimiento del tiempo de hemodiálisis

Uso frecuente de catéter venoso central 
de los encuestados. Asimismo, ha sido muy frecuente el incumplimiento en la medida del perfil férrico, recomendada cada 3-6 meses. Las GPC globales de mayor difusión en Latinoamérica y otras nacionales presentan diferentes criterios para iniciar el tratamiento con AEE y para definir el rango de $\mathrm{Hb}$ objetivo, pero todas coinciden en que $\mathrm{Hb}=12 \mathrm{~g} / \mathrm{dl}$ es el valor máximo a alcanzar con $A E^{6,11,12}$. Esta recomendación, que sigue vigente, se basa en los resultados de importantes estudios prospectivos controlados que evidencian mayor riesgo para los grupos tratados con AEE cuando se pretende alcanzar un nivel de $\mathrm{Hb}$ próximo al normal, mientras que los beneficios en la calidad de vida para los pacientes son mínimos o nulos ${ }^{13-17}$. Sorprende que más del $15 \%$ de los encuestados respondieran $13 \mathrm{~g} / \mathrm{dl}$ como valor máximo deseado. La encuesta no consultó específicamente acerca del conocimiento y aplicación de las GPC, pero estos resultados sugieren un escaso cumplimiento de éstas, aun cuando la población encuestada estaba constituida por nefrólogos (75.3\%), la mayoría de los cuales (55\%) tenían más de 10 años de trabajo en diálisis, lo que representa un grupo profesional de alta experiencia.

Este estudio muestra que el uso de AEE en Latinoamérica está sumamente extendido y que el FelV se indica a un porcentaje mucho menor de la población en diálisis. Pese a las diferencias metodológicas que limitan la comparación, estos resultados se parecen a los observados en registros basados en datos de pacientes de la región o a nivel internacional. Un estudio realizado tres años antes, con 9,025 pacientes latinoamericanos en HDC, mostró que el $84.3 \%$ de ellos recibían AEE y el $68.3 \%$, FelV 7 . Varios registros nacionales de 2013 muestran porcentajes similares: en Argentina, el $88.5 \%$ de los pacientes registrados usaban AEE; en Brasil, el $79 \%$ recibían AEE y el $53 \%$ usaban FelV; en Uruguay, el $80 \%$ recibían Epo y el 63\%, FelV ${ }^{18-20}$. La única distribución diferente la presentó Chile, donde el $67.8 \%$ de los pacientes en HDC recibían Epo y el 70\% usaban FelV ${ }^{21}$. Fuera de Latinoamérica, el informe Dialysis Outcomes and Practice Patterns Study (DOPPS) mostró que el $87-94 \%$ de los pacientes en HDC usaban AEE y el $67-85 \%$, FeIV, según datos de once países de tres continentes ${ }^{22}$. Esta coherencia con los resultados de otras publicaciones sobre el tema aporta validez a nuestra encuesta.

Nuestra encuesta detectó la existencia de pacientes con $\mathrm{Hb}$ baja que no recibían $\mathrm{AEE}$, y destaca que casi el $15 \%$ de los encuestados refirieron esa condición en más del $25 \%$ de esos pacientes.
El estudio previo también encontró que, pese al amplio uso de AEE, existía un $38.6 \%$ de la población en diálisis con $\mathrm{Hb}$ inferior a $10 \mathrm{~g} / \mathrm{dl}^{7}$. Esta realidad latinoamericana no coincide con la observada en otros países. Según datos del DOPPS Practice Monitor 2013, el $90 \%$ de los pacientes en HDC de EE.UU. recibían AEE y menos del $20 \%$ de toda la población presentaban $\mathrm{Hb}<10 \mathrm{~g} / \mathrm{dl}^{23}$. En Alemania, según la misma fuente, el $86 \%$ de los pacientes en HDC recibían AEE, mientras que menos del $10 \%$ de la población tenían $\mathrm{Hb}<10 \mathrm{~g} / \mathrm{dd}^{24}$. Las diferencias entre datos de países "desarrollados» y Latinoamérica para alcanzar el objetivo terapéutico mínimo de $\mathrm{Hb}$ en diálisis sugieren que las dificultades percibidas por nuestros encuestados pueden tener un papel causal, aunque seguramente éste no sea el único factor que explica el control insuficiente de la anemia.

Esta encuesta muestra una elevada frecuencia de dificultades para tratar la anemia de pacientes en diálisis, ya que el $72 \%$ de los colegas encuestados identificaron uno o más tipos de limitaciones. Encontramos una asociación significativa entre las respuestas que referían «mal control» de la anemia de pacientes tratados con AEE y la presencia de limitaciones para tratarlos. Asimismo existió una asociación entre las respuestas que reconocieron la existencia de pacientes con $\mathrm{Hb}$ baja que no recibían $\mathrm{AEE}$ y la presencia de limitaciones para tratar la anemia de su población.

El hallazgo de una asociación entre la presencia de limitaciones al tratamiento de la anemia y el grado de control de la misma, así como con el porcentaje de pacientes con $\mathrm{Hb}$ baja que no reciben $\mathrm{AEE}$, no permite asegurar relaciones causa-efecto, ya que esta encuesta no fue diseñada con ese fin. No obstante, creemos que esas limitaciones podrían relacionarse con factores socioeconómicos y la inequidad en el acceso a los recursos sanitarios; se necesitarían estudios más específicos para confirmarlo, así como su aparente impacto. En la población general, la asociación entre anemia y pobreza e inequidad ha sido bien estableci$\mathrm{da}^{25-27}$. Por otra parte, el RLADT ha comunicado una correlación significativa entre la prevalencia del tratamiento renal sustitutivo con el ingreso nacional bruto y otros indicadores de desarrollo humano de los países latinoamericanos, así que no sorprendería que existiera inequidad también en la disponibilidad de recursos para tratar la anemia de esa población ${ }^{28}$. No hemos encontrado referencias acerca de la asociación entre el tratamiento de la anemia de la ERC y las condiciones socioeconómicas y de los sistemas sanitarios, 
pero creemos que este estudio debería motivar un análisis específico al respecto.

Este estudio está basado en las respuestas de 513 médicos procedentes de 20 países latinoamericanos, nefrólogos o residentes en Nefrología en el $87.1 \%$ de los casos. La tasa de respuesta corresponde aproximadamente al $8 \%$ de los nefrólogos de Latinoamérica ${ }^{29}$. La distribución de los encuestados no se relaciona con el número de nefrólogos ni con la población de cada país, por lo que no existe representatividad por países. Su distribución se asocia, sin embargo, a la prevalencia de pacientes en diálisis crónica, según datos del RLADT, clasificada en tres grupos de países $^{29}$ :

- Los países con una prevalencia en diálisis superior a la media (> 468 pmp) (Argentina, Brasil, Chile, El Salvador, México, Puerto Rico y Uruguay) son representados por 311 encuestados (60.2\%).

- Los países con una prevalencia $\geq 250$ y $\leq 468$ pmp (Colombia, Cuba, Ecuador, Panamá, Perú y Venezuela) aportan respuestas de 155 médicos (30.2\%).

- Los países con una prevalencia inferior a 250 pmp (Bolivia, Costa Rica, Guatemala, Honduras, Nicaragua, Paraguay y República Dominicana) están representados por 47 colegas (9.2\%).

La principal limitación de esta encuesta, además de su carácter voluntario, es que recoge opiniones de médicos acerca del tratamiento de la anemia, sin contar con datos objetivos procedentes de los pacientes en diálisis.

La convocatoria alcanzada ha sido mayor que en el estudio previo con similares objetivos y ofrece conocimiento sobre una población más amplia, aunque tampoco resulta representativa por país?

La mayor fortaleza de la encuesta viene dada por la coherencia de sus resultados con los de otros estudios publicados, regionales e internacionales, así como por la consistencia interna de las respuestas, tanto entre sí como con los comentarios libres aportados. Esta consistencia nos ha permitido realizar cruzamientos válidos y analizar asociaciones entre variables.

\section{Conclusiones}

La encuesta confirma el extenso uso de AEE en HDC y DP, aunque sólo el $54 \%$ de los encuestados logran la $\mathrm{Hb}$ deseada en $\geq 75 \%$ de los pacientes tratados. Los criterios para indicar AEE, definir la $\mathrm{Hb}$ objetivo y controlar la anemia no son homogéneos, lo que evidencia el escaso cumplimiento de las GPC vigentes. Además, más del $70 \%$ de los encuestados refieren limitaciones para el estudio y/o tratamiento de la anemia de los pacientes en diálisis, y se observa cierto impacto en el resultado terapéutico. Este hallazgo, no obstante, debería reevaluarse con estudios diseñados específicamente a tal fin.

A pesar de las limitaciones de este estudio, creemos que los datos aportados brindan una visión global del manejo de la anemia de los pacientes en diálisis en nuestra región, de los recursos y las limitaciones existentes, que sugieren la elaboración de estudios más objetivos y específicos que permitan establecer los campos de mejora en este ámbito.

\section{Responsabilidades éticas}

Protección de personas y animales. Los autores declaran que para esta investigación no se han realizado experimentos en seres humanos ni en animales.

Confidencialidad de los datos. Los autores declaran que han seguido los protocolos de sus centros de trabajo sobre la publicación de datos de pacientes.

Derecho a la privacidad y consentimiento informado. Los autores declaran que en este artículo no aparecen datos de pacientes.

\section{Financiación}

Este estudio contó con el apoyo financiero de la SLANH.

\section{Conflicto de intereses}

Ninguno en relación con el tema abordado.

\section{Agradecimientos}

A todos los anónimos colegas participantes.

A la Comisión Directiva de la SLANH y a la Sra. Cinthya Uzcanegui, que colaboró desde su secretaría, así como a las autoridades de las sociedades miembro que colaboraron en la difusión de la encuesta.

Al Dr. Abayubá Pernas, experto uruguayo en estadística y epidemiología, por su asesoramiento en el manejo de los datos.

\section{Bibliografía}

\footnotetext{
1. Locatelli F, Pisoni RL, Combe C, Bommer J, Andreucci VE, Piera L, et al. Anaemia in five European countries and associated morbidity and mortality among haemodialysis patients: results from the Dialysis Outcomes and Practice Patterns Study (DOPPS). Nephrol Dial Transplant. 2004; 19(1):121-32.
} 
2. Johansen KL, Finkelstein FO, Revicki DA, Evans C, Wan S, Gitlin M, et al. Systematic review of the impact of erythropoiesis-stimulating agents on fatigue in dialysis patients. Nephrol Dial Transplant. 2012;27(6):2418-25.

3. McClellan W, Aronoff SL, Bolton WK, Hood S, Lorber DL, Tang KL, et al. The prevalence of anemia in patients with chronic kidney disease. Curr Med Res Opin. 2004;20(9):1501-10.

4. Astor BC, Muntner P, Levin A, Eustace JA, Coresh J. Association of Kidney Function With Anemia The Third National Health and Nutrition Examination Survey (1988-1994). Arch Intern Med. 2002;162(12):1401-8.

5. González-Bedat MC, Rosa-Diez G, Fernández Cean JM, Ordúñez $P$ Ferreiro A, Douthat W. National kidney dialysis and transplant registries in Latin America: how to implement and improve them. Rev Panam Salud Publica. 2015;38(3):254-60.

6. Comité Anemia de la SLANH, Carlini R, Andrade L, Bregman R, Campistrús MN, Chifflet $\mathrm{L}$, et al. Recomendaciones de práctica clínica de la Sociedad Latinoamericana de Nefrología e Hipertensión (SLANH) para el tratamiento de la anemia en el paciente con enfermedad renal crónica. Nefrología Latinoamericana. 2009;13(2):1-30.

7. Carlini R, Obrador G, Campistrús MN, Andrade L, Chifflet L, Bregman R, et al. The First Report of The Latin American Society of Nephrology and Hypertension (SLANH) Anemia Committee in Chronic Hemodialysis Patients. Nefrologia. 2014;34(1):96-104.

8. Rosa-Diez G, Gonzalez-Bedat M, Pecoits-Filho R, Marinovich S, Fernandez S, Lugon J, et al. Renal replacement therapy in Latin American end-stage renal Disease. Clin Kidney J. 2014;7(4):431-6.

9. Tessey A, Celia E, Ducasse E. Indicadores mínimos aplicables a pacientes prevalentes en tratamiento de diálisis en la República Argentina año 2014. Sociedad Argentina de Nefrología. [Internet]. Consultado en diciembre de 2016. Disponible en: http://san.org.ar/2015/docs/consensos/ pub_SAN_ACQ.pdf.

10. Fondo Nacional de Recursos. Programa de Presentación de Resultados. Uruguay año 2015. [Internet]. Consultado en julio de 2016. Disponible en: http://www.fnr.gub.uy/sites/default/files/resultados/dialisis_2015.pdf.

11. Kidney Disease: Improving Global Outcomes (KDIGO) Anemia Work Group. KDIGO Clinical Practice Guideline for Anemia in Chronic Kidney Disease. Kidney Int Suppl. 2012;2:279-335.

12. Documento de consenso sobre la enfermedad renal crónica. 27 de noviembre de 2012. [Internet]. Consultado en noviembre de 2016. Disponible en: http://www.senefro.org/modules/noticias/images/v._5.doc_ consenso_final_131212_copy1.pdf.

13. Besarab A, Bolton WK, Browne JK, Egrie JC, Nissenson AR Okamoto DM, et al. The effects of normal as compared with low hematocrit values in patients with cardiac disease who are receiving hemodialysis and epoetin. N Engl J Med. 1998;339(9):584-90.

14. Drueke TB, Locatelli F, Clyne N, Eckardt KU, Macdougall IC, Tsakiris D, et al. Normalization of hemoglobin level in patients with chronic kidney disease and anemia. N Engl J Med. 2006;355(20):2071-84

15. Singh AK, Szczech L, Tang KL, Barnhart H, Sapp S, Wolfson M, et al. Correction of anemia with epoetin alfa in chronic kidney disease. $\mathrm{N}$ Engl J Med. 2006;355(20):2085-98

16. Parfrey PS, Foley RN, Wittreich BH, Sullivan DJ, Zagari MJ, Frei D. Double-Blind Comparison of Full and Partial Anemia Correction in
Incident Hemodialysis Patients without Symptomatic Heart Disease. J Am Soc Nephrol. 2005;16(7):2180-9

17. Pfeffer MA, Burdmann EA, Chen CY, Cooper ME, de Zeeuw D, Eckardt KU, et al. Trial to Reduce cardiovascular Events with Aranesp Therapy (TREAT study). N Engl J Med. 2009;361(21):2019-32.

18. Marinovich S, Lavorato C, Bisigniano L, Celia E, Soratti C, Rosa Diez G, et al. Registro Argentino de Diálisis Crónica SAN-INCUCAI 2013. Sociedad Argentina de Nefrología e Instituto Nacional Central Único Coordinador de Ablación e Implante. Buenos Aires, Argentina, 2014. [Internet]. Consultado en noviembre de 2016. Disponible en: https://www.researchgate.net/publication/271504112_Registro_Argentino_de_Dialisis_Cronica_2013_Informe_2014.

19. Sesso RC, Lopes AA, Saldanha Thomé F, Lugon FR, Rinaldi dos Santos D. Inquérito Brasileiro de Diálise Crônica 2013 - Análise das tendências entre 2011 e 2013. J Bras Nefrol. 2014;36(4).

20. González Bedat C, Ferreiro Fuentes A, Schwedt E, Chifflet L. Informe Anual 2013 del Registro Uruguayo de Diálisis. [Internet]. Consultado en noviembre de 2016. Disponible en: http://nefrouruguay.com/wp-content/ uploads/2016/01/informeRUD2013_hemodialisis.pdf.

21. Poblete Badal H. 34. ${ }^{a}$ Cuenta de Hemodiálisis Crónica en Chile. Sociedad Chilena de Nefrología. [Internet]. Consultado en noviembre de 2016. Disponible en: http://www.nefro.cl/site/biblio/registro/11.pdf 2014.

22. 2012 DOPPS Annual Report. 2012 Annual Report of the Dialysis Outcomes and Practice Patterns Study: Hemodialysis Data 1997-2011. Arbor Research Collaborative for Health, Ann Arbor, MI. [Internet]. Consultado en noviembre de 2016. Disponible en: http://www.dopps.org/annualreport/.

23. DOPPS Practice Monitor: Trends in Anemia and MBD Management, and Interdialytic Weight Gain in US Hemodialysis Patients. DOPPS Practice Monitor. [Internet]. Consultado el 17 de diciembre de 2016. Disponible en: http://www.dopps.org/DPM/Default.aspx.

24. Reporting contemporary trends in German dialysis practice. DOPPS Practice Monitor. [Internet]. Consultado el 17 de diciembre de 2016. Disponible en: http://www.dopps.org/DPM/Germany/.

25. Benoist B, McLean E, Cogswell M, Egli I, Wojdyla D. Worldwide prevalence of anaemia 1993-2005. WHO Global Database on Anaemia. Ginebra, Suiza: World Health Organization; 2008.

26. Marín GH, Rivadulla P, Negro L, Gelemur M, Etchegoyen G; GIS. [Population study of the prevalence of anaemia in the adult population of Buenos Aires, Argentina]. Aten Primaria. 2008;40(3):133-8.

27. Vega Romero R, Acosta Ramírez N, Martínez Collantes J, Arrieta R, Estupiñán Z, Fonseca Z, et al. Análisis de disparidades por anemia nutricional en Colombia, 2005. Revista Gerencia y Políticas de Salud. 2008;7(15):46-76.

28. Cusumano AM, García García G, Gonzalez-Bedat MC, Marinovich S, Lugon J, Poblete Badal H, et al. Latin American Dialysis and Transplant Registry: 2008 prevalence and incidence of end-stage renal disease and correlation with socioeconomic indexes. Kidney Int Suppl (2011). 2013:3(2):153-6.

29. Rosa-Diez G, Gonzalez-Bedat M, Ferreiro A, García García G, Fernandez-Cean J, Douthat W. Burden of end-stage renal disease (ESRD) in Latin America. Clin Nephrol. 2016 Supplement 1;86 (2016)(13):29-33. 


\section{Anexo 1}

Encuesta sobre el control de la anemia en pacientes en diálisis crónica en Latinoamérica. Cuestionario

1. ¿Cuál es el nivel académico más alto que Usted ha alcanzado?

- Doctor en Medicina

- Residente de Medicina

- Residente de Nefrología

- Posgrado de Nefrología

- Otro (especifique cuál):

2. Género:

- Masculino

- Femenino

3. ¿Cuál es su categoría de edad?

Hasta 30 años

- De 31 a 40 años

- De 41 a 50 años

- De 51 a 60 años

- Más de 60 años

4. ¿En qué país de América Latina trabaja actualmente?

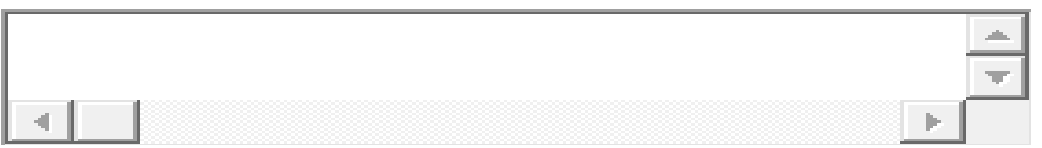

5. ¿Cuál es su principal ámbito laboral?

- Nefrología clínica

- Hemodiálisis

- Diálisis peritoneal

- Trasplante

- Investigación

- Administración

- Otro:

6. ¿Cuánto tiempo lleva trabajando en diálisis crónica?

- Menos de 1 año

- De 1 a 5 años

- De 5 a 10 años

- Más de 10 años

7. El centro de diálisis crónica donde usted trabaja corresponde a (responda lo que considere más adecuado):

- Hospital universitario

- Hospital público (no universitario)

- Hospital privado

- Centro de diálisis independiente

- Centro integrante de una empresa trasnacional

- Otro:

8. Actualmente ¿cuántos pacientes reciben tratamiento de diálisis crónica en la institución donde usted trabaja con mayor dedicación?

- Menos de 40

- De 40 a 70

- De 71 a 100 
- De 101 a 200

- $\quad$ Más de 200

9. ¿Qué tipo de tratamiento del agua se utiliza en el centro de hemodiálisis donde usted trabaja con mayor dedicación?

- Ósmosis inversa

- Desmineralizador en base a resinas

- Otro

- No conozco la respuesta

10. ¿Cuál es el nivel de aluminio del agua usada para diálisis en su centro durante el último año? Responda según el dato más reciente.

- Menos de 2 ug/l

- Menos de 5 ug/l

- Más de 5 y menos de 10 ug/l

- Más de 10 ug/l

- No se ha determinado aluminio en el último año

- No conozco ese dato

11. Aproximadamente, ¿qué porcentaje de los pacientes del servicio donde usted trabaja con mayor dedicación reciben eritropoyetina (u otro $\mathrm{AEE}$ )?

- Más del $90 \%$

- Entre el 75 y el $90 \%$

- Entre el 50 y el $75 \%$

- Menos del $50 \%$

- Otro comentario:

12. Aproximadamente, ¿qué porcentaje de los pacientes del Servicio de Hemodiálisis donde usted trabaja reciben FelV en forma habitual?

- Más del $90 \%$

- Entre el 75 y el $90 \%$

- Entre el 50 y el $75 \%$

- Menos del $50 \%$

Otro comentario:

13. Habitualmente, usted indica eritropoyetina ( $\mathrm{u}$ otro AEE) a sus pacientes en diálisis cuando su valor de Hb desciende de:

- $11 \mathrm{~g} / \mathrm{dl}$

$-10.5 \mathrm{~g} / \mathrm{dl}$

$-10 \mathrm{~g} / \mathrm{dl}$

$-\quad 9.5 \mathrm{~g} / \mathrm{dl}$

- Ninguno de los anteriores

Otro motivo (especifique cuál):

14. ¿Cuál es el nivel máximo de $\mathrm{Hb}$ que usted pretende que sus pacientes alcancen como respuesta al tratamiento con eritropoyetina?
$-11 \mathrm{~g} / \mathrm{dl}$
$-12 \mathrm{~g} / \mathrm{dl}$
$-13 \mathrm{~g} / \mathrm{dl}$
- Ninguno de los anteriores
- Variable para cada paciente
Otro (especifique cuál):

15. Aproximadamente, ¿qué porcentaje de sus pacientes tratados con eritropoyetina (u otro AEE) se halla en el rango de $\mathrm{Hb}$ esperado?

- Más del $90 \%$

- Entre el 75 y el $90 \%$ 
- $\quad$ Entre el 50 y el $75 \%$

- Menos del $50 \%$

16. ¿Qué porcentaje de sus pacientes portadores de $\mathrm{Hb}$ inferior al rango deseado no reciben eritropoyetina (u otro AEE) actualmente?

- Más del $50 \%$

- Entre el 25 y el $50 \%$

- Menos del $25 \%$

- Ninguno

17. ¿Con qué frecuencia mide la $\mathrm{Hb}$ en su centro de diálisis?

- Un vez al mes

- Una vez cada dos meses

- Una vez cada tres meses

- Variable (no se mide con regularidad periódica)

- Comentario:

18. ¿Cuál o cuáles de los siguientes parámetros utiliza usted habitualmente para evaluar el capital férrico de sus pacientes en hemodiálisis crónica? Varias respuestas posibles.

- Volumen corpuscular medio

- Sideremia (o ferremia)

- Índice de saturación de la transferrina

- Ferritina sérica

- Contenido de $\mathrm{Hb}$ reticulocitaria

- Porcentaje de glóbulos rojos hipocrómicos

- Hemoglobina corpuscular media

- Ninguno de los anteriores

- Otro (especifique cuál):

19. Aproximadamente, ¿qué porcentaje de sus pacientes en diálisis ha sido evaluado durante el último año con alguno de los siguientes parámetros?

\begin{tabular}{l} 
Volumen corpuscular medio \\
Sideremia (o ferremia) \\
IST \\
Contenitina sérica de $\mathrm{Hb}$ reticulocitaria \\
\hline Porcentaje de glóbulos rojos hipocrómicos
\end{tabular}

Comentario: 
20. ¿Qué tipo de limitaciones enfrenta usted para disponer de los recursos básicos para tratar la anemia de sus pacientes en diálisis? Varias respuestas posibles.

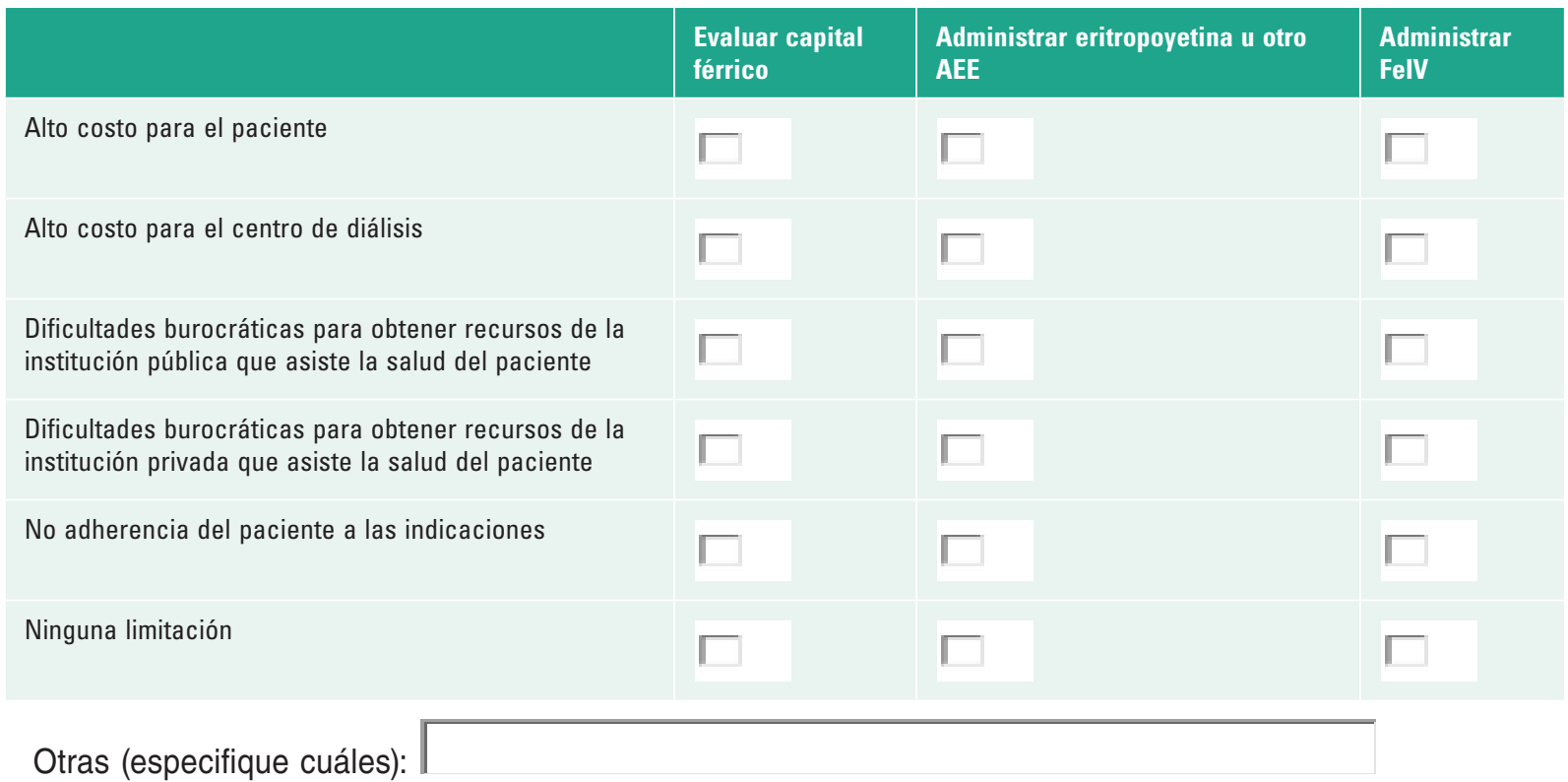

21. Por último, además de agradecer su tiempo, brindamos un espacio para agregar un comentario que considere pertinente:

Muchas gracias.

Comité de Anemia de la Sociedad Latinoamericana de Nefrología e Hipertensión 